\title{
GESTIÓN CADENA DE ABASTECIMIENTO - LOGISTICA CON INDICADORES BAJO INCERTIDUMBRE, CASO APLICADO SECTOR PANIFICADOR PALMIRA
}

\author{
SUPPLY CHAIN MANAGEMENT - LOGISTICS UNDER UNCERTAINTY \\ WITH INDICATORS, IF APPLIED PALMIRA PANIFICADOR CESTOR.
}

\begin{abstract}
Martín Darío Arango Serna
Ing. Industrial, Esp., M.Sc, Ph.D. Profesor Asociado Universidad Nacional de Colombia, Sede Medellín, Colombia. Investigador grupo GICO. mdarango@unalmed.edu.co
\end{abstract}

Wilson Adarme Jaimes

Ing. Industrial, Esp., M.Sc, Candidato a Doctor Universidad Nacional, Profesor asistente Universidad Nacional de Colombia, Sede Bogotá, Colombia. Investigador grupo SEPRO. wadarmej@unal.edu.co

Julían Andrés Zapata Cortes

Ing.Químico, Candidato a Magister en Ingeniería Administrativa Universidad Nacional de Colombia, Investigador grupo GICO UN Sede Medellín, Colombia. jazapat1@unalmed.edu.co

Fecha de recepción: 26 de febrero de 2010

Fecha de aprobación: 26 de abril de 2010

\section{RESUMEN}

Este artículo presenta el estudio de los sistemas de indicadores de desempeño de cadenas de abastecimiento en ambientes de incertidumbre. Se hace una distinción de los conceptos riesgo e incertidumbre, y se exponen las principales fuentes y estructuras de incertidumbre en cadenas de abastecimiento propuestas por diversos autores. Se mencionan las principales características de los indicadores de desempeño y se fusionan los dos elementos conceptuales (incertidumbre e indicadores de desempeño), para estudiar los modelos matemáticos que emplean los principios de la lógica difusa a fin de medir el desempeño de cadenas de abasto en ambientes de incertidumbre. Finalmente, se realiza una aplicación de un modelo en la cadena de abasto del sector panificador de Palmira. Los sistemas administrativo, talento humano y operativo fueron evaluados en 36 panaderías.

Palabras clave: indicadores, desempeño, incertidumbre, cadena de abastecimiento, lógica difusa. 


\section{ABSTRACT}

The main topics about performance indicators systems for supply chains under uncertainty environments are studied in this paper. A difference between risk and uncertainty concepts is made and are exposed the main uncertainty structures and sources in supply chains proposed by several experts' authors on the subject. Here is mentioned the main features of the performance indicators as a reflection of the management in supply chains and the above conceptual elements (uncertainty and performance indicators), in order to study the mathematical models that employs fuzzy logic principles to measure the performance of supply chains under uncertainty environments. Finally it is presented an application of a model in a company of the Palmira's Bread industry. The administrative, operational and human talents were evaluated in 30 bakeries.

Keywords: indicators, performance, uncertainty, supply chain, fuzzy logic.

\section{INTRODUCCIÓN}

El ambiente globalizado moderno cambió los actores tradicionales de la rivalidad empresarial, pasando de una competencia entre organizaciones individuales hacia una entre cadenas de abastecimiento, integradas por múltiples empresas proveedoras, manufactureras, distribuidoras o comercializadoras que garantizan el flujo del producto hasta el consumidor final.

Según el Foro Económico Mundial [1], el grado de sofisticación de las empresas en lo referente a la calidad de la gestión, a partir de las decisiones gerenciales tomadas en el entorno macroeconómico, explican el $28 \%$ de los resultados obtenidos en productividad y crecimiento de las mismas. El control de la gestión se apoya en indicadores, índices y cuadros productivos para ayudar a la organización a ser eficiente y efectiva en la captación y uso de los recursos operativos. Por ello, en la medida que se implementen técnicas y herramientas adecuadas con el entorno de acción empresarial, se puede garantizar un seguimiento del grado de cumplimiento de los objetivos. En el escenario mundial actual, para que las organizaciones alcancen algún grado de sostenibilidad mediante el continuo mejoramiento, las experiencias se deben apropiar y documentar. En este contexto, como lo indica Schhneeweiss [2], la oportunidad y la fiabilidad de la información constituyen un insumo básico para conocer la situación y posibilidades de las organizaciones.

La incertidumbre es una condición que está presente en todos los procesos industriales, dada la dificultad de predecir con precisión, por ejemplo, la demanda futura del bien o servicio o los tiempos de entrega de proveedores, que generalmente no es considerada para la toma de decisiones. El presente artículo busca entregar al lector, un componente teórico y conceptual acerca de dicha condición y cómo debe tratarse para evaluar las cadenas con indicadores de gestión. 
De acuerdo con la encuesta nacional de logística, año 2008, son contadas las empresas colombianas que utilizan sistemas de medición para evaluar su desempeño, en el cual la incertidumbre es un tema despreciado; en el presente estudio, se afronta la problemática que implica manejar e incluir la incertidumbre en los procesos de medición de desempeño de las cadenas de abastecimiento. Como referencia del trabajo, se tomaron las panificadoras de la ciudad de Palmira (Valle del Cauca), investigación que se ha venido adelantando desde el año 2002 hasta 2009, con financiación por parte de la Universidad Nacional, en la cual se ha identificado la necesidad de utilizar una adecuada gestión integral de la cadena de abasto, considerando el impacto que implica utilizar herramientas para modelar la incertidumbre en un ambiente donde la previsión, muchas veces es despreciada. El articulo presenta inicialmente la metodología, la situación general del contexto, una revisión de los indicadores de gestión en la cadena de abastecimiento; luego describe de manera breve, la teoría de los conjuntos difusos; posteriormente, presenta un ejemplo de aplicación, seguido de una discusión y las conclusiones.

\section{METODOLOGÍA}

El proceso metodológico que ha permitido entre otros productos, realizar este artículo, incluye una revisión teórica que se llevo a cabo durante los últimos años. Como es normal, en todas las industrias y en casi todas las operaciones reales, la incertidumbre juega un rol fundamental por no poder evitarse, lo cual conlleva a situaciones muchas veces indeseadas, en operaciones ejecutadas.

La Metodología consistió inicialmente, con una revisión exhaustiva de la importancia y necesidad de implementar la incertidumbre en los procesos de gestión mediante indicadores, pasando por la investigación de herramientas que permitieran incorporar dicha característica de los procesos, de donde se seleccionó la lógica difusa como una herramienta sencilla y aplicable al caso específico del sector de estudio.

La medición del desempeño de la cadena de suministro en la industria panificadora, está sujeta a gran variabilidad debido a la incertidumbre que genera el suministro y costo de materias primas, variación en los lead time, y falta de comunicación entre los escalones de la cadena entre otros. La investigación se inició en 2002, con una exploración de las organizaciones del subsector panificador en la zona urbana $\left(19.3 \mathrm{~km}^{2}\right)$, de Palmira, ciudad de 294.805 habitantes localizada en el sur del departamento del Valle del Cauca. De 202 panificadoras registradas en la Cámara de Comercio, 2006[3], 76 manifestaron receptividad para cooperar en la investigación. Teniendo en cuenta la disponibilidad de recursos, logística y conveniencia, se han caracterizado 36 panaderías. El estudio se desarrolló mediante la interacción de empresarios, trabajadores e investigadores, empleando herramientas como reuniones, encuestas, entrevistas semiestructuradas y observación participante del sistema. 


\subsection{SITUACION DEL SECTOR - PROBLEMÁTICA}

De acuerdo con la evaluación realizada por López y Lozano [4], el 3\% del total de establecimientos registrados en la ciudad, corresponde a panaderías que tienen en común, dificultades en su gestión y operación logística, motivadas entre otros aspectos, por la carencia de sistemas de información e indicadores apropiados para su gestión; $65 \%$ de las organizaciones tiene menos de cinco años de existencia, $57 \%$ tiene en promedio cuatro empleados (famiempresas). Adarme y Prieto [5], en la evaluación sobre competitividad del sector, prevén que la cooperación entre los artesanos del pan, sería un medio apropiado para mejorar la productividad, a partir de una gestión integrada de la cadena, adecuadas prácticas de almacenamiento y gestión de inventarios. Rivera y Villalobos [6], establecen las dificultades que tiene la implementación de las BPM, motivadas por las características de los medios que se utilizan en los procesos productivos (instalaciones, equipos, inventarios).

Estos estudios determinan que las dificultades apremiantes que presenta el sector, pasan por el manejo de inventarios (almacenamiento), acopio y distribución de insumos y disponibilidad de información sobre precios, transporte, competencia, proveedores, aplicación de normas e indicadores de gestión.

\subsection{INCERTIDUMBRE EN CADENAS DE ABASTECIMIENTO}

El estudio de la incertidumbre asociada a las cadenas de abastecimiento, se inicia desde la descripción de la misma, su percepción y por qué el conocimiento asociado al uso de tecnologías, sistemas de información y manejo de modelos matemáticos pueden ser fundamentales para su tratamiento. Es común que se usen indistintamente, los términos riesgo e incertidumbre para referirse a una condición desconocida de un evento futuro. El riesgo es una función de los resultados y su probabilidad, por lo cual puede ser estimado. Por el contrario, la incertidumbre se da cuando no se puede estimar el resultado de un evento o la probabilidad de su ocurrencia.

Establecida esta diferencia conceptual, se puede hacer una aproximación al estudio de la percepción y asociación de la incertidumbre en el ambiente de los negocios modernos, marcados por alta turbulencia y competencia en las cuales el recurso estratégico más importante para obtener ventaja competitiva es el conocimiento. En aquellas situaciones en las cuales la demanda es impredecible, los cambios tecnológicos rápidos y los ciclos de vida de los productos son cortos, una forma de obtener éxito en las operaciones es aplicando el conocimiento a los productos y a los procesos [7]. A medida que las empresas enfrentan mayor incertidumbre, mayores son los beneficios de aplicar conocimiento a la cadena de abastecimiento, y es clara una relación directa y positiva entre conocimiento y desempeño, a medida que aumenta la incertidumbre $[8,9]$. 
En el ámbito de las cadenas de abastecimiento, la incertidumbre es definida por Van Der Vorst y Beulens [10] así: "situaciones en las cuales el tomador de decisiones no sabe absolutamente qué decidir ya que está confundido respecto a los objetivos; carece de información acerca de la cadena de suministros y su ambiente; carece de información de las capacidades del proceso; no está en condiciones de predecir el impacto de las posibles acciones de control en el comportamiento de la cadena de suministro; o, carece de medidas eficaces de control".

La tipología de incertidumbre en cadenas de suministro, incluye tres dimensiones a cada fuente de incertidumbre, así [10]:

- Cantidad: cantidades de proveedores, demanda del cliente para la cantidad de producto, rendimiento y desperdicios de producto, disponibilidad de información.

- Calidad: calidad del proveedor, demandas de los clientes para las especificaciones de producto, calidad del producto después de la fabricación y almacenamiento y exactitud de la información.

- Tiempo: tiempo de entrega del proveedor, tiempo de distribución del pedido del cliente, tiempo de producción, tiempo de almacenamiento, tiempo de transferencia de la información, tiempo en tránsito.

Patel et al [11], establecen que la principal razón de incertidumbre en una cadena de abastecimiento es su dinamismo, y afirman que una cadena con múltiples niveles está sujeta a mayor grado de incertidumbre, debido a los grupos prestadores de servicios y a la existencia de incertidumbre en los elementos de cada nivel. Paulraj y Chen [12], consideran la demanda, la oferta y la tecnología como las tres fuentes de incertidumbre medioambientales en la administración de cadenas de abastecimiento.

Lee [13], propuso una estructura de incertidumbre medioambiental para cadenas de abastecimiento con cuatro tipos de incertidumbre: bajas incertidumbres de demanda y oferta; alta incertidumbre en la demanda y baja en la oferta; baja incertidumbre en la demanda y alta en la oferta, y altas incertidumbres de demanda y oferta. La incertidumbre de la demanda está vinculada a la capacidad de pronosticar la demanda de un producto. Según Fisher [14], los productos funcionales tienen demandas más predecibles y, por lo tanto, tienen bajas incertidumbres de demanda; a su vez, los productos innovadores tienen una demanda altamente impredecible.

Mason-Jones y Towill [15], adoptaron un enfoque de sistemas para detectar y codificar la incertidumbre en cadenas de suministro, basados en la identificación de problemas al estudiar los flujos a través de las interfaces de negocios, incluidos materiales, pedidos y flujos de caja y capacidad; desarrollaron el Modelo del Círculo de Incertidumbre (figura 1), para confirmar que ésta es un asunto estratégico en las cadenas de abastecimiento e identificaron sus cuatro principales fuentes: los suministros, el proceso de fabricación, 
los sistemas de control y la demanda. En este modelo, se enfatiza que la incertidumbre iniciada en los suministros y/o en los procesos de fabricación, puede ser mitigada con la aplicación de principios de gestión esbelta; y la incertidumbre causada por los sistemas de control y/o la demanda, requiere de la comprensión de la dinámica del sistema global.

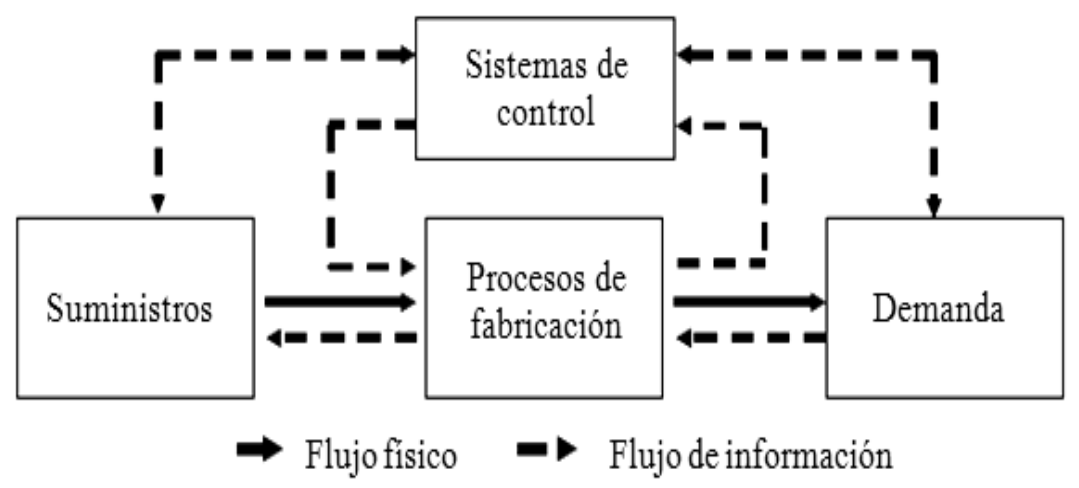

Figura 1. Modelo del Círculo de Incertidumbre. Fuente: (Mason-Jones y Towill, 1999).

Geary et al [16], desarrollaron una investigación en la industria automotriz, aplicando el enfoque del círculo de incertidumbre en el cual identificaron los principales tópicos asociados con los diferentes tipos de incertidumbre, al tratar de vincular las mejores prácticas de las cadenas de abastecimiento y los niveles de incertidumbre asociados a las mismas. Para combatir la incertidumbre y mejorar el desempeño propusieron una cadena de abastecimiento sin fisuras, que es un concepto ideal de flujos perfectos de información y materiales, facilitado por todos los actores de la cadena de abasto al actuar y pensar como uno solo.

Existe una serie de iniciativas colaborativas para enfrentar la incertidumbre y mejorar el desempeño de las cadenas de abastecimiento y la exactitud de los pronósticos. Para Bradley [17], un enfoque prometedor es el de Planeación, Pronóstico y Reaprovisionamiento Colaborativos (Collaborative Planning, Forecasting and Replenishment, CPFR), cuya idea principal es que los socios trabajen mancomunadamente en toda la cadena para determinar las estrategias de inventario y reaprovisionamiento, y realicen esfuerzos para alinear sus pronósticos de ventas consecuentemente. Si la iniciativa es exitosa, se logra disminución de inventarios en toda la cadena, aumento de la rotación de los mismos, disminución del agotamiento de existencias y aumento de la rentabilidad. La implementación de iniciativas de este tipo, requiere de un soporte tecnológico que garantice la conectividad de los socios y la rastreabilidad de los productos. 


\subsection{INDICADORES DE DESEMPEÑO DE CADENAS DE SUMINISTROS}

Los indicadores de desempeño son unos instrumentos para monitorear la consecución de los objetivos de la cadena de abastecimiento. La información que proporcionan debe ser siempre de naturaleza cuantitativa (incluso si se miden variables cualitativas), y dirigida a un diagnóstico de la situación para mejorar. El instrumento de medida por aplicar, debe ser simple, sencillo y fácil de interpretar, teniendo en cuenta la interdependencia de las actividades de la cadena de abastecimiento, su elaboración y publicación, y debe ser orientado lo más objetivamente posible, a un auténtico control de gestión.

Hugos [18], establece la necesidad en las empresas de identificar el mercado en donde se encuentran como una característica fundamental para el correcto desempeño de la información recolectada y entregada por los indicadores. También afirma que este mercado está definido por las condiciones de oferta y demanda, y que puede encontrarse cuatros tipos del mismo: en desarrollo, en crecimiento, estable y maduro.

El mercado en desarrollo corresponde a aquél en el cual hay un lanzamiento de nuevos productos o el desarrollo de un nuevo mercado; en este caso tanto la oferta como la demanda son bajas; los mercados en crecimiento son aquellos en las cuales la demanda es mayor que la oferta y, por ende, el suministro es incierto; en un mercado estable, la demanda y el suministro son altos y, por lo tanto, relativamente predecibles; $y$, en el mercado maduro, el suministro excede ampliamente la demanda. Sin embargo, tal como se mencionó anteriormente, las condiciones de competencia generan una incertidumbre asociada a la demanda de los productos y servicios.

En la tabla 1, se esquematizan estos tipos de mercado y su comportamiento con la demanda y la oferta.

Tabla 1. Clasificación de mercados.

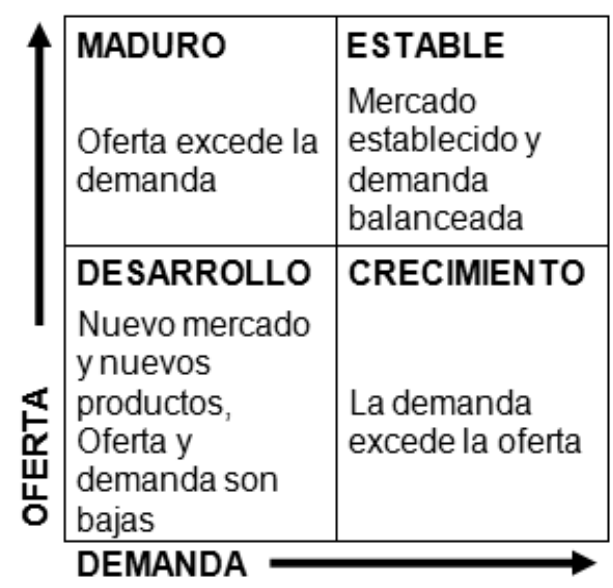

Fuente: Hugos, 2003 
Hugos [18], afirma que una vez que las empresas identifican los mercados, deben recoger la información más importante sobre los mismos, que permita entender el grado de respuesta al mercado y guiar el comportamiento de la organización. Para ello, las empresas deben realizar medidas del desempeño en cuatro categorías: servicio al cliente, eficiencia interna, flexibilidad a la demanda y desarrollo de productos. Conociendo estas categorías y el mercado en donde la empresa interactúa, es posible disponer de un marco conceptual que permite evaluar el desempeño de la compañía.

Tabla 2. Medidas de desempeño

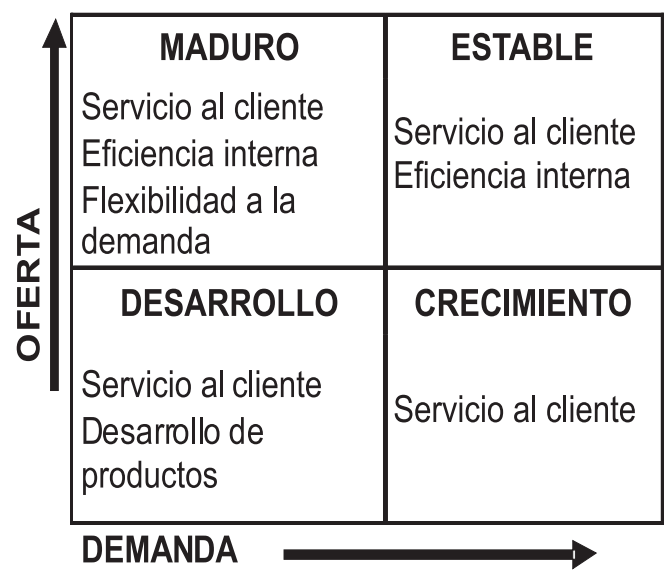

Fuente: Hugos, 2003

Cada mercado requiere un grupo específico de elementos para evaluar. En la tabla 2, se observan las categorías de desempeño para evaluar en cada uno de los mercados en donde se encuentre la empresa. Las medidas de desempeño más importantes en las cadenas de suministros para cada una de las categorías son:

\section{Servicio al cliente:}

- Para mercados de alta demanda y volumen de producción: relación de cumplimiento de órdenes, relación de entrega a tiempo, relación de valor y número de pedidos rechazados o en espera por falta de productos, frecuencia y duración de pedidos rechazados o en espera por falta de productos, y relación de devoluciones.

- Para mercados especializados de productos hechos a medida: tiempo de respuesta a órdenes, relación de entregas a tiempo, valor de entregas tarde y relación de las mismas, frecuencia y duración de entregas tardías, número de productos devueltos por garantía, y número de reparaciones.

\section{Eficiencia interna:}

- Valor del inventario, Rotación de inventario, Ingresos sobre ventas, Ciclo de caja. 


\section{Flexibilidad a la demanda:}

- Tiempo de ciclo de actividades, Capacidad de responder a aumentos súbitos de la demanda (Upside Flexibility), Capacidad de responder a pedidos de productos fuera de línea o nuevos (Outside Flexibility).

\section{Desarrollo de productos:}

- $\quad$ Porcentaje total de ventas de productos introducidos en el último año

- Porcentaje de SKU's totales introducidos en el último año

- Tiempo de ciclo para el desarrollo de nuevos productos y entrega de los mismos.

Por otra parte, Anaya [19], considera que en el diseño de indicadores logísticos de gestión, se debe considerar los siguientes aspectos:

- Definición inequívoca y aceptabilidad: todo indicador debe estar perfectamente definido en cuanto a su contenido y fórmula de cálculo, y debe relacionarse con un objetivo concreto, perfectamente cuantificable, entendido y aceptado por los responsables.

- Modo de expresión: los indicadores deben expresarse en forma de proporciones o porcentajes que son más estables que las cifras absolutas, fáciles de interpretar y permiten analizar la evolución de las magnitudes, así como la comparación con otros indicadores similares de otros sectores o departamentos.

- Nivel de agregación: es conveniente que los indicadores se instrumenten en un determinado nivel de agregación definido, como familias de productos, sector de mercado, etc.

- Simplicidad operativa: los datos deben ser sencillos de calcular e interpretar, empleando en lo posible, herramientas informáticas que actúen sobre bases de datos fiables y actualizados.

- Factibilidad: los objetivos previstos deben ser realistas y realizables, tomando siempre como base la situación actual.

- Presentación: deberá ser siempre de forma gráfica, comparando la evolución con relación al objetivo propuesto, centrándose en la tendencia y no en situaciones puntuales poco significativas.

Una de las tantas tipologías de indicadores de desempeño, se puede clasificar en cualitativos y cuantitativos.

\subsection{INDICADORES DE DESEMPEÑO CUALITATIVOS}

Son aquellos para los cuales no existe medida numérica directa, aunque algunos aspectos pueden ser cuantificados. En esta categoría, se encuentran entre otros, los siguientes indicadores, según Chan [20]: 
- Satisfacción del cliente: grado con el cual los clientes son satisfechos con el producto o servicio recibido.

- Flexibilidad: capacidad de la cadena de abastecimiento para responder a las fluctuaciones aleatorias en los patrones de demanda.

- Integración de los flujos de materiales e información: grado en el cual todas las funciones en la cadena de abastecimiento, pueden intercambiar información y materiales sin inconvenientes.

- Gestión efectiva del riesgo: grado en el cual se minimizan los efectos de los riesgos.

- $\quad$ Desempeño de proveedores: mide el desempeño de los proveedores de materias primas en cuanto a calidad de las mismas y tiempo de entrega.

\subsection{INDICADORES DE DESEMPEÑO CUANTITATIVOS}

Son aquellos que pueden ser medidos numéricamente en forma directa. Pueden subdividirse en cuatro clases así, según Nyhuis [21]:

- Indicadores de costos o financieros: costos de la cadena de abastecimiento completa, ingresos por ventas, utilidades, inversión en inventarios, el rendimiento de la inversión.

- Indicadores de respuesta al cliente: tasa de cumplimiento, porcentaje de entregas tardías, tiempo de respuesta al cliente, tiempo de pedido, duplicación de funciones.

- Indicadores de productividad: porcentaje de utilización de la capacidad instalada, porcentaje de utilización de los recursos.

- Indicadores de calidad: mide la efectividad en la ejecución de las actividades o procesos, aportando resultados sobre el número de errores cometidos, así como de entregas perfectas y sin errores.

\subsection{INDICADORES DE DESEMPEÑO EN AMBIENTES DE INCERTIDUMBRE}

El ambiente de incertidumbre propio de las cadenas de abastecimiento supone ciertas limitaciones al momento de implementar sistemas de indicadores de desempeño de las mismas bajo el enfoque de métodos exactos o determinísticos, dado que éstos requieren conocer con exactitud el valor de los datos que alimentan el sistema. Según Petrovic et al [22], tradicionalmente, la literatura ha modelado los parámetros de incertidumbre en problemas propios de gestión de cadenas de abastecimiento, usando distribuciones de probabilidad que se derivan de las evidencias registradas en el pasado. Sin embargo, cuando no se posee dicha evidencia o falta certidumbre sobre la misma, 
los métodos probabilísticos estándar no son apropiados. En este caso, los parámetros de incertidumbre se especifican con base en la experiencia y el juicio subjetivo del tomador de decisiones.

La teoría de conjuntos difusos proporciona una estructura apropiada para describir y tratar la incertidumbre relacionada con la imprecisión. Por ejemplo: se puede expresar la demanda en términos de vaguedad y representarla en conjuntos difusos con distribuciones de posibilidad asociadas.

Lau et al [23], consideran que es posible utilizar la lógica difusa para resolver situaciones complejas en cadenas de suministros analizando el desempeño de sus integrantes, y desarrollaron una metodología para analizar y monitorear el desempeño de los proveedores en una cadena de suministros, basados en los criterios de calidad de producto y tiempo de entrega. Los factores desconocidos para la construcción de un indicador global de desempeño fueron tratados, usando el enfoque de lógica difusa. El resultado del proceso de inferencia es un conjunto con una distribución difusa como respuesta. Sin embargo, dado que generalmente se utilizan respuestas puntuales como insumo para la toma de decisiones, en cuanto a cantidad de bienes por ordenar de un proveedor específico, se necesita eliminar la difusidad para obtener un número discreto. Existen varios métodos para eliminar la difusidad como el del Centro de Gravedad y Media de los Máximos; el método adecuado depende del grado de ajuste del mismo, a las consideraciones y restricciones de la cadena de abastecimiento.

Chan et al [20], propusieron un modelo matemático que emplea la teoría de conjuntos difusos para medir el desempeño integral de una cadena de abastecimiento en ambientes de incertidumbre (figura 2). Inicialmente se obtienen los datos de los indicadores de desempeño adecuados, los cuales son ponderados, encontrando el peso normalizado de su importancia la escala geométrica de un número difuso triangular; la asignación de estos pesos relativos está a cargo de un Equipo de Medición del Desempeño, conformado por personal de todas las áreas de la organización, con capacitación y experiencia en el tema. Luego, se define un grado difuso de desempeño para encontrar el resultado difuso de la medición y calcular la calificación del desempeño; la determinación del intervalo de la escala de desempeño (nivel aceptable hasta nivel totalmente satisfactorio), se hace contrastando los objetivos establecidos en la planeación con los registros históricos y el entorno donde se lleva a cabo la operación. Finalmente, se elimina la difusidad de los resultados de los indicadores, para obtener un indicador que permita ser usado para reflejar el desempeño de la cadena de suministro en evaluación. 


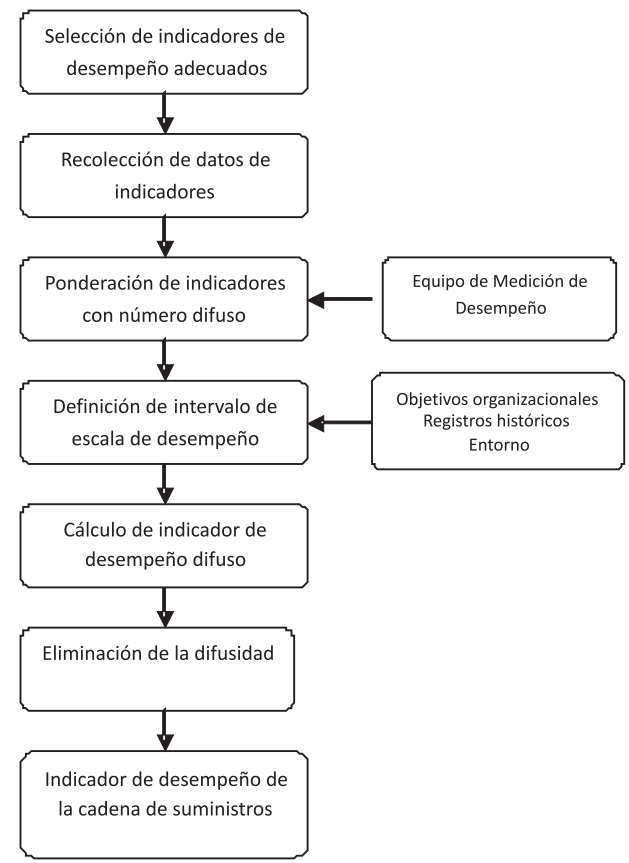

Figura 2. Procedimiento del modelo de medición de desempeño de cadenas de suministros usando lógica difusa

Fuente: Adaptado de Chan et al, 2003

Dado lo anterior y con el fin de dar claridad al modelo desarrollado en este articulo, se ha considerado que la lógica difusa es una herramienta modelizadora apropiada para evaluar matemáticamente algunas de sus variables en condiciones de incertidumbre.

\section{RESULTADOS Y DISCUSIÓN}

A partir de la caracterización que se ha hecho de los indicadores de gestión, y previendo la evaluación en un contexto real, se ha tomado como referencia aquel sector que el grupo de investigación viene trabajando desde hace ocho años (sector de alimentos panaderías), y para mostrar la aplicabilidad de la lógica difusa en el mismo, se presentan indicadores relevantes de la investigación.

Para el estudio, el grupo investigador según criterios propios, clasificó a las panaderías en tres categorías así: grandes (PG), aquellas que tienen más de diez empleados; medianas (PM), entre cinco y diez empleados, y pequeñas (PP), las que tienen entre uno y cuatro empleados. 
Tabla 3. Características las Panaderías. Caso de estudio.

\begin{tabular}{|c|c|c|c|}
\hline CARACTERÍSTICA & $\begin{array}{l}\text { PANADERÍAS } \\
\text { PEQUEÑAS }\end{array}$ & $\begin{array}{l}\text { PANADERÍAS } \\
\text { MEDIANAS }\end{array}$ & PANADERÍAS GRANDES \\
\hline $\begin{array}{l}\text { La estructura } \\
\text { organizativa está } \\
\text { integrada, así: }\end{array}$ & $\begin{array}{l}\text { El } 76 \% \text { por los } \\
\text { subsistemas de } \\
\text { producción y } \\
\text { ventas. } \\
\text { El } 65 \% \text { no tiene } \\
\text { organigrama }\end{array}$ & $\begin{array}{l}\text { El } 30 \% \text { por los } \\
\text { subsistemas de } \\
\text { producción y } \\
\text { ventas. } \\
30 \% \text { tienen } \\
\text { organigrama }\end{array}$ & $\begin{array}{l}\text { El } 34 \% \text { dispone de cuatro } \\
\text { subsistemas (producción, } \\
\text { ventas, contabilidad, } \\
\text { administrativo) } \\
\text { El } 33 \% \text { tiene dos subsistemas } \\
\text { (producción y ventas) } \\
\text { El } 33 \% \text { tiene tres subsistemas } \\
\text { (producción, ventas y } \\
\text { contabilidad) } \\
\text { El } 67 \% \text { tiene organigrama }\end{array}$ \\
\hline $\begin{array}{c}\text { La organización, } \\
\text { gestión, y } \\
\text { satisfacción de las } \\
\text { necesidades de los } \\
\text { clientes }\end{array}$ & $\begin{array}{l}\text { El } 88 \% \text { manifestó } \\
\text { que cumple }\end{array}$ & $\begin{array}{l}80 \% \text { manifestó que } \\
\text { cumple }\end{array}$ & El $100 \%$ manifestó que cumple \\
\hline $\begin{array}{l}\text { Documentación } \\
\text { organizacional }\end{array}$ & $\begin{array}{l}\text { El } 65 \% \text { no posee } \\
\text { misión ni visión } \\
\text { explícita. } \\
\text { No posee manual } \\
\text { de funciones ni } \\
\text { reglamento interno } \\
\text { de trabajo. } \\
\text { El } 18 \% \text { dispone de } \\
\text { manual de higiene y } \\
\text { seguridad industrial }\end{array}$ & $\begin{array}{l}\text { El } 70 \% \text { ha } \\
\text { explicitado la } \\
\text { misión y visión } \\
10 \% \text { posee manual } \\
\text { de funciones. } \\
\text { El } 30 \% \text {, reglamento } \\
\text { interno de trabajo. } \\
\text { El } 40 \% \text { manual de } \\
\text { higiene y seguridad } \\
\text { industrial }\end{array}$ & $\begin{array}{l}\text { El } 100 \% \text { tiene misión y visión. } \\
\text { El } 66 \% \text { posee manual de } \\
\text { funciones y reglamento interno } \\
\text { de trabajo. } \\
\text { El } 34 \% \text {, manual de higiene y } \\
\text { seguridad industrial }\end{array}$ \\
\hline Permisos & $\begin{array}{l}71 \% \text { tiene registro } \\
\text { de Industria y } \\
\text { Comercio. } \\
77 \% \text { tiene licencia } \\
\text { de saneamiento. }\end{array}$ & $\begin{array}{l}\text { El } 90 \% \text { tiene } \\
\text { registro de industria } \\
\text { y comercio. } \\
\text { El } 70 \% \text { tiene } \\
\text { licencia de } \\
\text { saneamiento. }\end{array}$ & $\begin{array}{l}\text { El } 100 \% \text { tiene registro de } \\
\text { industria y comercio, y posee } \\
\text { licencia de saneamiento }\end{array}$ \\
\hline Área & $\begin{array}{l}\text { El } 40 \% \text { tiene entre } \\
60-80 \mathrm{~m}^{2} . \\
\text { El } 40 \% \text { tiene áreas } \\
\text { hasta de } 120 \mathrm{~m}^{2}\end{array}$ & $\begin{array}{l}\text { El } 60 \% \text { tiene entre } \\
120-160 \mathrm{~m}^{2}\end{array}$ & El $60 \%$ tiene entre $150-200 \mathrm{~m}^{2}$ \\
\hline Maquinaria & $\begin{array}{l}\text { Tiene entre } 11 \text { y } 15 \\
\text { máquinas. } \\
\text { Los equipos más } \\
\text { comunes son: } \\
\text { horno, horno } \\
\text { giratorio, mojadora, } \\
\text { cilindro y batidora. }\end{array}$ & $\begin{array}{l}\text { Un } 80 \% \text { tiene entre } \\
16 \text { y } 20 \text { máquinas. } \\
\text { Mantenimiento } \\
\text { correctivo. } \\
\text { Los equipos más } \\
\text { comunes son: } \\
\text { horno, horno } \\
\text { giratorio, mojadora, } \\
\text { cilindro y batidora. }\end{array}$ & $\begin{array}{l}\text { El } 100 \% \text { tiene entre } 16 \text { y } 20 \\
\text { máquinas. } \\
\text { Tratándose de equipos flexibles } \\
\text { frente al volumen y la variedad } \\
\text { de productos, el mantenimiento } \\
\text { es preventivo. } \\
\text { Los equipos más comunes son: } \\
\text { horno, horno giratorio, } \\
\text { mojadora, cilindro y batidora. }\end{array}$ \\
\hline $\begin{array}{l}\text { Insumos (harina de } \\
\text { trigo, azúcar, queso, } \\
\text { sal, levadura, } \\
\text { margarina) - anual } \\
\text { agregado }\end{array}$ & $\begin{array}{l}2.316 \text { toneladas, } \\
\text { con un promedio de } \\
16 \text { toneladas. }\end{array}$ & $\begin{array}{l}468 \text { toneladas, con } \\
\text { un promedio por } \\
\text { panadería de } 22 \\
\text { toneladas. }\end{array}$ & $\begin{array}{l}336 \text { toneladas, con un } \\
\text { promedio por panadería de } 38 \\
\text { toneladas. }\end{array}$ \\
\hline $\begin{array}{l}\text { Ocupación promedio } \\
\text { de hombres por } \\
\text { panadería }\end{array}$ & 3 & 6 & 12 \\
\hline
\end{tabular}


La relación hombre a hombre entre Panaderías Grandes y Panaderías Pequeñas es de 4:1, mientras que por consumo de insumos está en una relación de Panaderías Grandes a Panaderías Pequeñas de 2:4:1. La aparente mayor productividad de la mano de obra en las Panaderías Pequeñas, obedece en parte a la indefinición de horarios, salarios, incentivos y demás elementos integrantes de la relación laboral, por tratarse de la unidad familiar nuclear o ampliada (padres, hijos, primos, sobrinos y demás miembros de la familia).

La investigación ha establecido los rasgos e indicadores sobresalientes del subsector panificador de Palmira, los cuales permiten a empresarios, consumidores, proveedores, clientes, autoridades e instituciones, disponer de información necesaria e importante para tomar decisiones, dependiendo del usuario de la misma. Para el grupo investigador, esta información es un insumo primario en el compromiso de apoyo a la industria y permite trabajar en el diseño de estrategias y acciones para continuar con la exploración de alternativas que beneficien en conjunto, a los diferentes actores que participan en el sector, particularmente en el diseño de modelos y/o aplicativos "hechos a la medida" de cada organización, soportados en sus antecedentes, trayectoria y proyección, aunado a los desarrollos que se vienen realizando desde la academia.

La caracterización ha generado la necesidad de continuar con una investigación aplicada que atienda los factores relevantes que podrían estar incidiendo en los niveles actuales de competitividad del sector; en tal sentido, el grupo de investigación destaca la necesidad y el compromiso de investigar en el diseño de una red de distribución de insumos para la cadena de abastecimiento del subsector, que incluya la gestión de los inventarios con criterios de justo a tiempo, en un ambiente de Inventario Gestionado por el Vendedor (Vendor Managed Inventory, VMI), y el diseño de la red que determine la mejor localización del centro de acopio y distribución, que articule los esfuerzos de los artesanos del pan, que ofrezcan alternativas concretas para tratar la situación de manejo, transporte y almacenamiento de las materias primas. De esta forma, se espera continuar con la exploración de alternativas que faciliten la sostenibilidad de los microempresarios en el sector.

A continuación en este aparte de resultados y discusión se presenta como hecho relevante, la aplicación de lógica difusa para tratar indicadores en escenarios bajo incertidumbre como son los que presenta el sector de alimentos.

Una vez seleccionado el modelo y la herramienta para enfrentar la incertidumbre, se hizo una aplicación de los mismos en la medición del desempeño de la cadena de abastecimiento de las empresas panificadoras de Palmira, teniendo como criterio de medición, el tiempo de respuesta a las órdenes de pedidos hechas a los proveedores (lead time). Históricamente, este indicador se tiene en un promedio de 12 horas, desde que se posiciona una orden hasta que ésta llega a la panificadora. 
Sin embargo, al realizar el estudio, se encontró que los tiempos histórico y actual de este indicador, varían entre 10 y 14 horas durante los 12 meses del año. Se seleccionó un Equipo de Medición del Desempeño integrado por un representante de las diferentes áreas funcionales (operarios, supervisores y dirección de operaciones). Estos evaluadores asignaron los pesos relativos $W=(0,13.0,35.0,52)$, al indicador, de acuerdo con sus consideraciones personales y profesionales, teniendo presente su conocimiento y experiencia en el sector, lo cual en calidad de expertos definen estos pesos como los más indicados para esta evaluación.

El primer evaluador consideró como meta, reducir el tiempo a 9 horas, por lo cual la puntuación difusa del desempeño sería:

$$
P d_{1}=\left(\frac{(14-10) *(10-0)}{(14-9)}\right)=8
$$

La determinación de los grados de desempeño difuso se hace, teniendo en cuenta la distribución triangular mostrada en la figura 3.

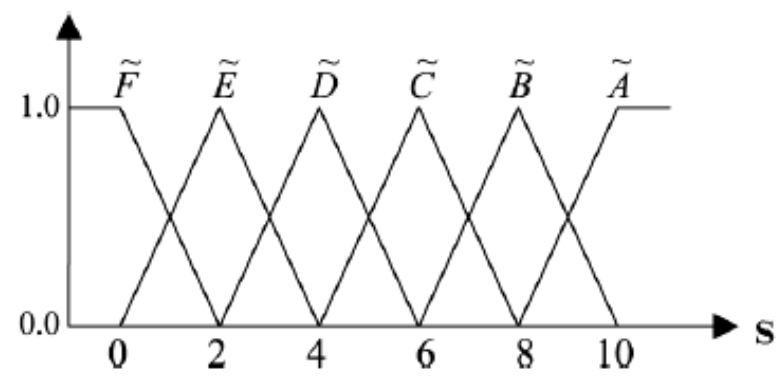

Figura 3. Grado difuso triangular

Fuente: Chan et al, 2003

Así, los grados de desempeño difuso individuales obtenidos son los siguientes:

$$
\begin{aligned}
& P_{A}(3,5)=0 \\
& P_{B}(3,5)=1 \\
& P_{C}(3,5)=0 \\
& P_{D}(3,5)=0 \\
& P_{E}(3,5)=0 \\
& P_{F}(3,5)=0
\end{aligned}
$$

Luego, el conjunto difuso que expresa el grado de desempeño obtenido según el criterio del primer evaluador, es el siguiente:

$$
P_{1}=(0.1 .0 .0 .0 .0)
$$


El segundo evaluador consideró como meta, reducir el tiempo a 8 horas y el tercero, a 7,5 horas. La puntuación difusa del desempeño para cada uno de ellos es:

$$
\begin{aligned}
& P d_{2}=6,67 \\
& P d_{3}=5,71
\end{aligned}
$$

Utilizando el mismo procedimiento realizado con el primer evaluador, los conjuntos difusos resultantes para expresar el desempeño según los criterios del segundo y tercer evaluador son:

$$
\begin{gathered}
P_{2}=(0 \cdot 0,37 \cdot 0,63 \cdot 0 \cdot 0 \cdot 0) \\
P_{3}=(0 \cdot 0 \cdot 0,8 \cdot 0,2 \cdot 0 \cdot 0)
\end{gathered}
$$

La matriz del grado de desempeño difuso resultante es:

$$
P=\left[\begin{array}{ccc}
0 & 0 & 0 \\
1 & 0,37 & 0 \\
0 & 0,63 & 0,8 \\
0 & 0 & 0,2 \\
0 & 0 & 0 \\
0 & 0 & 0
\end{array}\right]
$$

El resultado consolidado de la medición del indicador por parte de los tres evaluadores, está dado por la multiplicación de la matriz anterior por el vector $W=(0,13 ; 0,35 ; 0,52)$, correspondiente a los pesos asignados inicialmente por los evaluadores:

$$
P * W=[0.0,2595.0,6365.0,104.0 .0]
$$

La eliminación de la difusidad del indicador global difuso anterior, permite obtener un valor puntual del Indicador de Desempeño (ID):

$$
\begin{gathered}
I D=\left(10^{*} 0+8^{*} 0,2595+6^{*} 0,6365+4^{*} 0,104+2^{*} 0+0 * 0\right) /(0+0,2595+0,6365+ \\
0,104+0+0) \\
I D=6,31
\end{gathered}
$$

Este resultado se compara en una escala de 0 a 10; por lo tanto, se puede afirmar que las organizaciones en estudio, tienen un desempeño del $63.1 \%$ en el indicador de lead time, basado en el criterio de los evaluadores seleccionados.

Un aspecto fundamental en la aplicación de este modelo para la medición del desempeño de la cadena de abastecimiento en ambientes de incertidumbre, es la escogencia del Equipo de Medición de Desempeño, dado que los resultados por obtener dependen en gran manera del razonamiento individual de cada uno de ellos, producto de su 
conocimiento sobre el funcionamiento de toda la cadena de abastecimiento y todos los factores que lo determinan.

\section{CONCLUSIONES}

El conocimiento del desempeño, expresado en los indicadores, es clave para lograr el compromiso del personal en los resultados organizacionales, por lo cual el plan de indicadores debe incluir una política clara y específica de divulgación a todos los niveles de la organización, garantizando su asimilación y entendimiento, específicamente, haciendo notar cómo las labores que el personal realiza en forma cotidiana, afectan los resultados globales. De esta forma, las panificadoras de Palmira podrán ejecutar de forma adecuada, la gestión y medición del desempeño de su actividad en busca de altos niveles de competitividad.

La implementación de un sistema de indicadores de desempeño para el control de la gestión en cadenas de abastecimiento, debe estar soportada en sistemas de información confiables que faciliten la toma de decisiones en forma oportuna, especialmente cuando se trabaja con estrategias de coordinación colaborativas para la disminución de la incertidumbre; de lo contrario, es necesario incluir esta condición de vaguedad en el análisis con los indicadores, tal como es el caso de las panaderías del sector panificador de Palmira. El éxito de un plan de indicadores de desempeño no es proporcional al número de indicadores que se imponga. Por el contrario, se recomienda empezar sólo con unos pocos bien seleccionados que sean adecuados a las características propias de la organización y el sector de negocios.

Existen modelos matemáticos para medir el desempeño de cadenas de suministros en ambientes de incertidumbre, los cuales son herramientas que ayudan al mejoramiento de las operaciones mediante la evaluación de indicadores de gestión que consideran los factores más relevantes de dichas cadenas.

La aplicación de los principios de la lógica difusa en el proceso de diseño y ejecución de indicadores de desempeño de cadenas de abastecimiento pone en concomitancia situaciones reales del discernimiento humano con la vaguedad propia de las actividades de medición de gestión en el ambiente empresarial actual, ya que se puede enfrentar de manera más adecuada, la solución de problemas de incertidumbre de la vida real y no usando los enfoques probabilísticos tradicionales.

Las limitaciones de la aplicación de la lógica difusa, en la evaluación del desempeño de cadenas de abastecimiento en ambientes de incertidumbre, están relacionadas con la definición de las reglas difusas del sistema por modelar, dado que se requiere de la opinión de expertos en resultados experimentales o derivaciones teóricas. 


\section{REFERENCIAS BIBLIOGRÁFICAS}

[1] Foro Económico Mundial. Informe anual: (2007) Global Competitiveness Report.

[2] Schneeweiss, CH. (2003) Distributed decision making--a unified approach. Europ J Operat Res 150 pp. 237-252.

[3] Cámara de Comercio de Palmira (2006). Anuario estadístico.

[4] López A., Lozano G. (2004). Caracterización técnica y diagnóstico del subsector panificador de Palmira. Trabajo de grado. (Administrador de Empresas). Universidad Nacional. Palmira.

[5] Adarme W., Prieto R. (2006). Competitividad en el subsector panificador de Palmira. (Trabajo Especial). Universidad del Valle. 65p.

[6] Rivera C., Villalobos L. (2006). Caracterización de las pymes subsector agroalimentario de la ciudad de Palmira y municipios aledaños en función de las Buenas Prácticas de Manufactura BPM y el sistema de análisis de peligros y puntos críticos de control HACCP. Proyecto de grado (Administradoras de Empresas). Universidad Nacional de Colombia.

[7] Grant R., (1996) Prospering in dynamically-competitive environments: Organizational capability as knowledge integration. Organization Science, Vol. 7, № 4, pp. 375-387.

[8] Grant R. and Baben-Fuller C. (1995) Knowledge based theory of inter-firm collaboration. Academy of Management Conference.

[9] Claycomb C., Droge C. and Germain R. (2001) Applied process knowledge and market performance: the moderating effect of environmental uncertainty. Journal of Knowledge Management, Vol. 5, Nㅜ 3, pp. $264-278$.

[10] Van Der Vorst J. and Beulens A. (2002) Identifying sources of uncertainty to generate supply chain redesign strategies. International Journal of Physical Distribution and Logistics Management, Vol. 32, №6, pp.409-430.

[11] Patel C., Gunasekaran A., and Mcgaughey, R. (2004) A framework for supply chain performance measurement. International Journal of Production Economics, Vol. 87, pp. 333-347.

[12] Paulraj A. and Chen I.J. (2007) Environmental uncertainty and strategic supply management: a resource dependence perspective and performance implications. The Journal of Supply Chain Management, Vol. 43, № 3, pp. 29-42. 
[13] Lee H. (2002) Aligning supply chain strategies with product uncertainties. California Management Review, Vol. 44, №3, pp. 105-119.

[14] Fisher M. (1997) What is the right supply chain for your product? Harvard Business Review, Vol. 75, № 2, pp. 105-116.

[15] Mason-Jones R. and Towill D.R. (1999) Shrinking the supply chain uncertainty circle. Control, The Institute of Operations Management, Vol. 24, №7, pp.17-22.

[16] Geary S., Childerhouse P., and Towill D. (2002) Uncertainty and the seamless supply chain. Supply Chain Management Review, July-August, pp. 52-61.

[17] Bradley P., (2001) The certainty of uncertainty. Supply Chain Management Review, Vol. $1, \mathrm{~N}^{\circ} 3$.

[18] Hugos M., (2003) Essentials of supply chain management. New Jersey: John Wiley \& Sons. pp. 137-170.

[19] Anaya J., (2007) Logística integral, la gestión operativa de la empresa. 3 ed. Madrid: ESIC. 290 p.

[20] Chan F. et al. (2003) A conceptual model of performance measurement for supply chains. Management Decision, Vol. 41, № 7, pp. $635-642$.

[21] Nyhuis P., (2007) Fundamentals of production logistics: Theory, Tools and Applications. 2 ed. Londres: Springer. 300 p.

[22] Petrovic D. et al. (1999) Supply chain modeling using fuzzy sets. International Journal of Production Economics, Vol. 59, pp. 443 - 453.

[23] Lau H. et al. (2002) Methodology for monitoring supply chain performance: a fuzzy logic approach. Logistics Information Management, Vol. 15, N, pp. $271-280$. 
